\title{
Singular Values of Differences of Positive Semidefinite Matrices ${ }^{1}$
}

\author{
Xingzhi Zhan \\ Graduate School of Information Sciences, Tohoku University
}

\section{Introduction}

Let $M_{n}$ be the space of $n \times n$ complex matrices. For simplicity we treat matrices here, but all our results hold for compact operators on a Hilbert space. Suppose $A, B \in M_{n}$ are positive semidefinite. We shall study the relations between the singular values of

$$
A-B \text { and }\left(\begin{array}{cc}
A & 0 \\
0 & B
\end{array}\right)
$$

and those of

$$
A-|z| B, \quad A+z B, \quad \text { and } \quad A+|z| B
$$

where $z$ is a complex number.

A norm $\||\cdot|||$ on $M_{n}$ is called unitarily invariant if $\|U A V\||\|=\| A \||$ for all $A$ and all unitary $U, V$. Every unitarily invariant norm is a symmetric gauge function of the singular values. See $[3,7]$. We always denote the singular values of $A$ by $s_{1}(A) \geq \cdots \geq s_{n}(A)$, and put $s(A) \equiv\left(s_{1}(A), \ldots, s_{n}(A)\right)$. Familiar examples of unitarily invariant norms are the Ky Fan $k$-norms defined by $\|A\|_{(k)}=\sum_{1}^{k} s_{j}(A)$ and the Schatten $p$-norms: $\|A\|_{p}=\left(\sum_{1}^{n} s_{j}^{p}(A)\right)^{1 / p}$, $p \geq 1$. Note that $\|\cdot\|_{\infty}$ is just the operator (spectral) norm and $\|\cdot\|_{2}$ is the Frobenius norm.

\footnotetext{
${ }^{1}$ This paper is to appear in SIAM J. Matrix Anal. Appl.
} 
A unitarily invariant norm may be considered as defined on $M_{n}$ for all orders $n$ by the rule

$$
\|A\| \mid=\left\|\left(\begin{array}{cc}
A & 0 \\
0 & 0
\end{array}\right)\right\| \|
$$

i.e., adding or deleting zero singular values does not affect the value of the corresponding symmetric gauge function.

Given a real vector $x=\left(x_{i}\right) \in \mathbb{R}^{n}$, rearrange its components as $x_{[1]} \geq \cdots \geq$ $x_{[n]}$. For $x=\left(x_{i}\right), y=\left(y_{i}\right) \in \mathbb{R}^{n}$, if

$$
\sum_{1}^{k} x_{[i]} \leq \sum_{1}^{k} y_{[i]}, \quad k=1,2, \ldots, n
$$

then we say $x$ is weakly majorized by $y$, denoted $x \prec_{w} y$. If the components of $x$ and $y$ are nonnegative and

$$
\prod_{1}^{k} x_{[i]} \leq \prod_{1}^{k} y_{[i]}, \quad k=1,2, \ldots, n
$$

we say $x$ is weakly log-majorized by $y$, denoted $x \prec_{w l o g} y$. See [6] for a discussion of this topic.

Denote the block diagonal matrix $\left(\begin{array}{cc}A & 0 \\ 0 & B\end{array}\right)$ by $A \oplus B$. Bhatia and Kittaneh [4, Remark 5] observed that if $A, B \in M_{n}$ are positive semidefinite then

$$
\|A-B\||\leq\|A \oplus B\||
$$

for every unitarily invariant norm. By the Fan dominance principle [3, 7], (1.1) is equivalent to $s(A-B) \prec_{w} s(A \oplus B)$. We shall show that in fact each singular value of $A-B$ is not greater than the corresponding singular value of $A \oplus B$.

In another paper, Bhatia and Kittaneh [5, Thm 1] proved that for positive semidefinite $A, B \in M_{n}$ and any complex number $z$

$$
|||A-| z|B||| \leq|||A+z B||| \leq|||A+| z|B|||
$$


for all unitarily invariant norms. Again (1.2) is equivalent to

$$
s(A-|z| B) \prec_{w} s(A+z B) \prec_{w} s(A+|z| B)
$$

We shall prove that the corresponding weak log-majorizations hold. Since weak log-majorization implies weak majorization [6,7], our result strengthens (1.2).

\section{Main Results}

Our first result sharpens (1.1).

Theorem 1 Let $A, B \in M_{n}$ be positive semidefinite. Then

$$
s_{j}(A-B) \leq s_{j}(A \oplus B), \quad j=1,2, \ldots, n .
$$

The following result sharpens (1.2).

Theorem 2 Let $A, B \in M_{n}$ be positive semidefinite. Then for any complex number $z$

$$
s(A-|z| B) \prec_{w \log } s(A+z B) \prec_{w l o g} s(A+|z| B) .
$$

The special case $z=i=\sqrt{-1}$ of Theorem 2 says

$$
s(A-B) \prec_{w l o g} s(A+i B) \prec_{w l o g} s(A+B) .
$$

It has been proved in [2] that for positive $A, B$ and $p>1$

$$
s\left(A^{p}+B^{p}\right) \prec_{w} s\left((A+B)^{p}\right) .
$$


When $p \geq 2$, the above relation is refined as follows:

$$
s\left(A^{p}+B^{p}\right) \prec_{w} s\left(\left(A^{2}+B^{2}\right)^{p / 2}\right) \prec_{w} s\left(|A+i B|^{p}\right) \prec_{w l o g} s\left((A+B)^{p}\right) .
$$

The first relation in (2.5) follows from (2.4) and the third relation follows from (2.3). To see the second relation let $T=A+i B$. This is the Cartesian decomposition. From $A^{2}+B^{2}=\left(T^{*} T+T T^{*}\right) / 2$ we get

$$
s\left(A^{2}+B^{2}\right) \prec_{w} s\left(|A+i B|^{2}\right) .
$$

Note that $f(t)=t^{p / 2}$ is convex and increasing on $[0, \infty)$. By a majorization principle [3, 7], applying this $f$ to the preceding weak majorization yields the second relation in (2.5).

From (2.3) and the results in [1] and [2] it follows that for $0<p \leq 1$,

$$
\begin{array}{ccc}
s\left(A^{p}-B^{p}\right) \prec_{w} s\left(|A-B|^{p}\right) \prec_{w l o g} s\left(|A+i B|^{p}\right) & \prec_{w l o g} & s\left((A+B)^{p}\right) \\
& \prec_{w} & s\left(A^{p}+B^{p}\right) .
\end{array}
$$

One might wonder whether the weak majorization (2.4) can be replaced by the stronger log-majorization. The answer is no, even for $p=2$. Consider the example

$$
A=\left(\begin{array}{ll}
1 & 0 \\
0 & 0
\end{array}\right), \quad B=\left(\begin{array}{ll}
1 & 1 \\
1 & 1
\end{array}\right) .
$$

We have $\operatorname{det}\left(A^{2}+B^{2}\right)=2>1=\operatorname{det}\left[(A+B)^{2}\right]$.

Recently we have generalized Theorem 1 and the second majorization result in Theorem 2 to the case of $\tau$-measurable operators affiliated with a semifinite von Neumann algebra.

Acknowledgement This work was done while the author was at the Graduate School of Information Sciences, Tohoku University as a postdoctoral fellow of 
the Japan Society for the Promotion of Science. He thanks JSPS for the support and Professor F. Hiai for helpful discussions.

\section{REFERENCES}

1. T. Ando, Comparison of norms ||$|f(A)-f(B)| \|$ and $\||f(|A-B|)|\|$, Math. Z., 197(1988) 403-409.

2. T. Ando and X. Zhan, Norm inequalities related to operator monotone functions, Math. Ann., 315(1999) 771-780.

3. R. Bhatia, Matrix Analysis, Springer, 1997.

4. R. Bhatia and F. Kittaneh, On the singular values of a product of operators, SIAM J. Matrix Anal. Appl., 11(1990) 272-277.

5. R. Bhatia and F. Kittaneh, Norm inequalities for positive operators, Letters Math. Phys., 43(1998) 225-231.

6. F. Hiai, Log-majorizations and norm inequalities for exponential operators, in Linear Operators, Banach Center Publications, Vol. 38, 1997, pp.119-181

7. R. A. Horn and C. R. Johnson, Topics in Matrix Analysis, Cambridge University Press, New York, 1991. 